\title{
Hydrogen bonding aggregation in acrylamide: theory and experiment
}

\author{
Elena Patyukova, ${ }^{*, \dagger}$ Taylor Rottreau, ${ }^{\dagger}$ Robert Evans, ${ }^{\dagger}$ Paul D. Topham, ${ }^{*}{ }^{\dagger}$ and \\ Martin J. Greenall*,‡ \\ $\dagger$ Aston Institute of Materials Research, Aston University, Birmingham, B4 7ET, UK \\ $\ddagger$ School of Mathematics and Physics, University of Lincoln, Brayford Pool, Lincoln, LN6 \\ 7TS, UK \\ E-mail: patyukova@gmail.com; p.d.topham@aston.ac.uk; mgreenall@lincoln.ac.uk
}




\begin{abstract}
Hydrogen bonding plays a role in the microphase separation behavior of many block copolymers, such as those used in lithography, where the stronger interactions due to hydrogen bonding can lead to a smaller period for the self-assembled structures, allowing the production of higher resolution templates. However, current statistical thermodynamic models used in descriptions of microphase separation, such as the FloryHuggins approach, do not take into account some important properties of hydrogen bonding, such as site specificity and cooperativity. In this combined theoretical and experimental study, a step is taken toward the development of a more complete theory of hydrogen bonding in polymers, using polyacrylamide as a model system. We begin by developing a set of association models to describe hydrogen bonding in amides. Both models with one association constant and two association constants are considered. This theory is used to fit IR spectroscopy data from acrylamide solutions in chloroform, thereby determining the model parameters. We find that models with two constants give better predictions of bond energy in the acrylamide dimer and more realistic asymptotic behavior of the association constants in the limit of high temperatures. At the end of the paper, we briefly discuss the question of the determination of the Flory-Huggins parameter for a diblock copolymer with one self-associating hydrogen-bonding block and one non-hydrogen-bonding block by means of fitting the scattering function in a disordered state.
\end{abstract}

\title{
Introduction
}

Hydrogen bonding interactions occur very widely in nature. Although individual bonds are relatively weak, their effect on the physical properties of substances can be profound, and is responsible for the anomalous properties of water and the secondary structure of proteins. However, the characteristics of hydrogen bonding, such as site specificity and cooperativity, make it difficult to build a general theoretical description of H-bonding systems ${ }^{1}$. 
One of the most natural ways to describe the thermodynamics of the formation of hydrogen bonds is to treat this phenomenon as a reversible chemical reaction. This association model approach (sometimes called the ERAS model ${ }^{2}$ ) was initially proposed to describe hydrogen bonding association in alcohols and polyalcohols ${ }^{3}$. In the framework of this model, it is assumed that alcohols in the liquid state form a full range of linear chain aggregates due to hydrogen-bonding association. It was shown that chemical equilibrium in this kind of system is described well by two association constants, one corresponding to the formation of a dimer (dimer association constant) and the other corresponding to addition of further molecules to the chain (multimer association constant).

The association model of hydrogen bond formation in alcohols was later applied by Painter and Coleman to describe the miscibility of hydrogen-bonding homopolymers ${ }^{3,4}$. They showed that association constants measured for low molecular weight analogs of polymer segments can be used (after rescaling in order to take into account the difference between the molar volumes of monomers and polymer segments) to describe hydrogen bonding in polymer systems. This approach has several strengths: (i) its parameters are measurable quantities, (ii) it treats non-hydrogen-bonding interactions and hydrogen-bonding interactions separately, (iii) the number of hydrogen bonded contacts is not random, and (iv) it works as an extension of the Flory-Huggins theory of polymer melts, which is the basic theoretical platform in polymer physics ${ }^{5}$. However, as this work was focused specifically on alcohols, hydrogen bonding in homopolymers and diblock copolymers is still often described by means of a negative Flory-Huggins parameter ${ }^{6-8,9}$.

Taking into account the virtues of the association model approach, it is useful to extend it to other classes of self-associating hydrogen-bonding compounds such as amides and acids. Here, we develop a set of association models for amides and test them by comparison with IR absorption measurements on acrylamide solutions.

The choice of acrylamide as a system to study is motivated by the current interest in the properties of its corresponding polymer, polyacrylamide. Polyacrylamide is a commercially 
important polymer which, in addition to its uses in chromatographic columns, soft contact lenses and cosmetics, is now finding applications in the areas of biomaterials and smart materials research ${ }^{10,11}$. A key factor in these applications is hydrogen bonding: the acrylamide group has both hydrogen donor and acceptor sites and can serve as a universal hydrogen bonding agent.

In addition to the strengths of the association model listed above, we also believe that it will yield insights into hydrogen-bonded acrylamide aggregates that would be difficult, or even impossible, to obtain by other techniques, such as density functional theory (DFT) ${ }^{12,13}$ and molecular dynamics simulations ${ }^{14}$. DFT is a powerful tool that gives many valuable insights into the physics of hydrogen bonding, such as the effect of the conformation and relative positions of the molecules on the energy of the hydrogen bonding interaction. It can also be used to investigate hydrogen-bonded clusters, and such studies have been carried out for acetamide ${ }^{15,16}$. However, to the best of our knowledge, existing studies on acrylamide focus on the structure and spectral features of isolated molecules and hydrogen-bonded dimers and do not provide any information on networks of hydrogen bonds or on the entropy of hydrogen bond formation in an ensemble of molecules.

Molecular dynamics (MD) has also been used to study hydrogen-bonded networks and, for example, has been used to investigate liquid formamide ${ }^{14}$. However, there are questions about the use of MD in these systems, since it was shown in the case of alcohols that molecular dynamics simulations using common force fields do not reproduce the spectral features of hydrogen-bonded aggregates in solution, even on a qualitative level ${ }^{17}$. Furthermore, there is always a constraint on the size of the system in molecular dynamics simulations, which limits the possibility of simulating the true size distribution of aggregates in solution.

The main difficulty in constructing an association model of acrylamide is that there is not enough information about the "rules" of association and the minimal number of association constants necessary to describe association. In the literature of the interpretation of IR data from solutions of amides, all models that we are aware of use linear chain aggregates or 
cyclic dimers ${ }^{18}$. In the crystal phase, acrylamide is known to form two-dimensional ribbonlike networks of hydrogen bonds ${ }^{19}$ with two bonds per oxygen, two bonds per $\mathrm{NH}_{2}$ group and ribbons built from the double-bonding of cyclic dimers. However, we believe that, in both the solution and the melt, the association model may be different. For example, for relatively large acetamide clusters with aggregation numbers up to $i=15^{16}$, it was shown by means of DFT simulations that clusters with "irregular" (as well as linear and cyclic) structure have lower energies than clusters constructed from crystal polymorphs, and we expect similar behavior for acrylamide.

Our strategy to deal with the this uncertainty is to develop a set of models with different association rules. We start with models with one association constant (in other words, in all these models we assume that all hydrogen bonds have the same energy regardless of their position inside the aggregate). These models are then applied to interpret IR spectroscopy data from acrylamide solutions in chloroform and determine the association constants. Next, models with two association constants are investigated. These give better correspondence to the bond energies in acrylamide dimers predicted by $\mathrm{DFT}^{12,13}$.

At the end of the paper, we take a first step toward the application of the association model approach to the description of hydrogen bonding in block copolymers by calculating the structure factor of a disordered melt of block copolymer with one hydrogen-bonding selfassociating block and one non-hydrogen-bonding block in the random phase approximation (RPA). Next, the models and parameters determined for acrylamide are used to estimate how the Flory-Huggins parameter that appears in the RPA formula for the structure factor of the diblock copolymer with a polyacrylamide block is shifted by the presence of hydrogen bonds in these systems. It should be noted that these results can be considered only as preliminary, because, though our calculations take into account the non-randomness of hydrogen bonding contacts, they do not take into account the non-randomness of mixing in polymer systems, which should be accounted for in the future. 


\section{Models with one association constant}

According to one definition, a hydrogen bond is an attractive short-ranged force between a hydrogen atom bonded to a strongly electronegative atom and another electronegative atom with a lone pair of electrons.

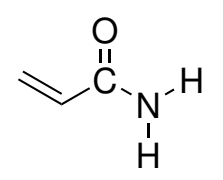

Figure 1: Acrylamide molecule.

Acrylamide has a primary amide group (see Figure 1), which consists of an oxygen atom (a hydrogen acceptor) and an $\mathrm{NH}_{2}$ group (a hydrogen donor). The oxygen atom can potentially form two hydrogen bonds as it has two lone electron pairs. Since it has two hydrogens, the $\mathrm{NH}_{2}$ group can also potentially form two bonds. However, it is also possible that the oxygen atom will predominantly form only one bond (as in alcohols) ${ }^{20}$ or that the second hydrogen on the $\mathrm{NH}_{2}$ group will lose its donating properties after the first hydrogen becomes bonded ${ }^{21}$. Different rules of association produce aggregates of different architectures. If we allow only one bond per oxygen and one bond per $\mathrm{NH}_{2}$ group, then the hydrogen-bonding association of acrylamide molecules produces linear chains. In all other cases, branched aggregates are produced.

From both experimental studies (for example, NMR spectroscopy) and DFT simulations it is known that tautomerism is not present in acrylamide because imidic acid has an energy approximately $11 \mathrm{kcal} / \mathrm{mol}$ higher than the ground state energy of the syn-isomer ${ }^{13}$, so we do not consider the possibility of tautomerism in our models. Isomerism is also not considered, because $95 \%$ of the molecules are in the syn-isomer state at room temperature due to the large difference in ground state energy between the two isomers ${ }^{22}$. In all of our models, it is also assumed that the two hydrogens are equivalent and the slight difference in electronegativity between them is neglected.

In this section, we consider models for which it is assumed that all hydrogen bonds have 
the same energy regardless of their position in the aggregate and that there are no cycles of any kind. The list of models generated by these assumptions and different association rules is presented in Table 1.

Table 1: List of models with one association constant and no cycles.

\begin{tabular}{|l|l|}
\hline Model & association rules \\
\hline \hline $\mathbf{0}$ & only linear dimers \\
\hline $\mathbf{1}$ & one bond per oxygen, two bonds per $\mathrm{NH}_{2}$ group \\
\hline $\mathbf{2}$ & two bonds per oxygen, two bonds per $\mathrm{NH}_{2}$ group \\
\hline $\mathbf{3}$ & one bond per oxygen, one bond per $\mathrm{NH}_{2}$ group \\
\hline $\mathbf{4}$ & two bonds per oxygen, one bond per $\mathrm{NH}_{2}$ group \\
\hline
\end{tabular}

In order to illustrate our calculation method, model 1 is used as an exemplar. The calculations for all other models can be found in the supplementary materials.

In model 1, it is assumed that the rules of association can be formulated as "one bond per oxygen, two bonds per $\mathrm{NH}_{2}$ group". The starting assumption is that the free energy of the solution can be written as

$$
F=F_{0}+F_{\mathrm{HB}}
$$

where $F_{0}$ is the free energy of solution without any hydrogen bonding association of the dissolved molecules and $F_{\mathrm{HB}}$ is the contribution due to hydrogen bond formation.

If there are $N$ acrylamide molecules in solution with $M$ hydrogen bonds in total between them and the energy of one hydrogen bond is $\epsilon$, one can write ${ }^{23}$

$$
F_{\mathrm{HB}}=M \epsilon-k T \ln \left[p^{M} \Xi\right],
$$

where $p$ is the probability of formation of one bond, which can be expected to be inversely proportional to the volume of the system, so that $p=C / V$ (where $C$ is a constant), and $\Xi$ is the combinatorial number of ways to form $M$ bonds in the system. It is worth noting that here we implicitly use our assumption about the absence of cycles because we suppose that the formation of each bond contributes to the statistical weight factor $p$, which accounts 
for the loss of entropy due to bond formation. In the case when an additional bond is formed that leads to the creation of a cycle, the entropy loss is either absent or much smaller because the participating molecules are already held close to each other by other bonds in the aggregate.

The expression for $\Xi$ in the case when one bond per oxygen and two bonds per $\mathrm{NH}_{2}$ group is allowed has the form

$$
\Xi=\frac{N ! 2^{M}}{(N-M) !} \frac{(2 N-2) !}{(2 N-2-M) !} \frac{1}{M !} .
$$

Here, the first factor is the number of ways to choose $M$ acceptors for $M$ bonds out of $N$ molecules, taking into account that each oxygen can form only one bond, but has two bonding sites. The second factor is the number of ways to choose $M$ donors for the bonds (at this stage all atoms are treated as distinguishable) and the last factor accounts for the indistinguishability of bonds. Since $M$ and $N$ are large numbers, the -2 terms in the second factor will be neglected.

Substituting $\Xi$ into Equation 2, using Stirling's formula and minimizing $F_{\mathrm{HB}}$ with respect to $M$ yields

$$
\frac{M}{2(N-M)(2 N-M)}=\frac{K}{V},
$$

where the equilibrium association constant $K \equiv C \exp \left(-\frac{\epsilon}{k T}\right)$ has been introduced. Alternatively, in terms of concentrations

$$
\frac{m}{2(n-m)(2 n-m)}=K \text {. }
$$

Solving this quadratic equation with respect to $m$, the dependence of the free energy on the total concentration of solution can be obtained as

$$
\frac{F_{\mathrm{HB}}}{k T V}=m+n \ln \left[\frac{(n-m)(2 n-m)^{2}}{4 n^{3}}\right] \text {, }
$$


where

$$
m=\frac{1+6 K n-\sqrt{1+12 K n+4 K^{2} n^{2}}}{4 K} .
$$

In order to couple this expression with the Flory-Huggins formula for the free energy of the system without hydrogen bonds, it can be rewritten in terms of volume fractions as

$$
\frac{F_{\mathrm{HB}} v}{k T V}=\phi_{m}+\phi \ln \left[\frac{\left(\phi-\phi_{m}\right)\left(2 \phi-\phi_{m}\right)^{2}}{4 \phi^{3}}\right]
$$

with

$$
\phi_{m}=\frac{1+6 K^{\prime} \phi-\sqrt{1+12 K^{\prime} \phi+4 K^{\prime 2} \phi^{2}}}{4 K^{\prime}}
$$

where $v$ is a reference volume and $K^{\prime}=K / v$ is a dimensionless association constant. As the calculation method is the same for all models, only the final expressions for the free energies of the other models with one association constant are presented here, in Table 2. The full derivations can be found in the Supporting Information.

Table 2: Free energies for models with one association constant.

\begin{tabular}{|l|c|c|}
\hline Model & $F_{\mathrm{HB}} v / k T V$ & $\phi_{m}$ \\
\hline \hline 0 & $2 K^{\prime} \phi_{1}^{2}+\phi \ln \frac{\phi_{1}}{\phi}$ & $\frac{1+16 K^{\prime} \phi-\sqrt{1+32 K^{\prime} \phi}}{32 K^{\prime}}$ \\
\hline $1 \& 4$ & $\phi_{m}+\phi \ln \left[\frac{\left(\phi-\phi_{m}\right)\left(2 \phi-\phi_{m}\right)^{2}}{4 \phi^{3}}\right]$ & $\frac{1+6 K^{\prime} \phi-\sqrt{1+12 K^{\prime} \phi+4 K^{\prime 2} \phi^{2}}}{4 K^{\prime}}$ \\
\hline 2 & $\phi_{m}+4 \phi \ln \left(1-\frac{\phi_{m}}{2 \phi}\right)$ & $\frac{1+4 K^{\prime} \phi-\sqrt{1+8 K^{\prime} \phi}}{2 K^{\prime}}$ \\
\hline 3 & $\phi_{m}+2 \phi \ln \left(1-\frac{\phi_{m}}{\phi}\right)$ & $\frac{1+8 K^{\prime} \phi-\sqrt{1+16 K^{\prime} \phi}}{8 K^{\prime}}$ \\
\hline
\end{tabular}

Model 4 is similar to model 1 but with the roles of donors and acceptors exchanged, and is therefore described by the same equations. However, in this model, the number of free $\mathrm{NH}_{2}$ groups will be different from model 1, so it is considered as a separate case here.

\section{Determination of association constants}

The association constants of alcohols were previously measured by others using IR spectroscopy with the help of the following idea ${ }^{24,25}$. Suppose that the hydrogen-bonding sub- 
stance is dissolved in a solvent that has no specific (i.e. hydrogen bonding or strong polar) interactions with the solute. Then, at vanishingly small concentrations of solute, peaks corresponding to the vibrations of the hydrogen-bonding groups in isolated molecules should be seen. As the concentration is increased, new peaks should appear that correspond to hydrogen-bonded states of hydrogen-bonding groups, as hydrogen bonding changes the absorption frequency of groups participating in the bond. In consequence, the dependence of the height of the peaks corresponding to absorption by isolated molecules should have a weaker than linear dependence on the total concentration of the solution. So, if a formula can be found to describe how the concentration of the species corresponding to a given peak depends on the total concentration, then the association constants can be determined by fitting this expression to experimental results on the dependence of the peak height on the total concentration, with the association constants treated as adjustable parameters.

This procedure is now applied to the case of solutions of acrylamide in chloroform. Chloroform is chosen because it is a non-hydrogen bonding solvent that dissolves acrylamide sufficiently well to give a good range of concentrations (compared, for example, to carbon tetrachloride), and because it has a relatively high boiling temperature (compared, for example, to dichloromethane), to allow the measurements to be conducted over a sufficiently broad range of temperatures.

Figure 2 shows the changes of IR absorption by acrylamide in the range $3700 \mathrm{~cm}^{-1}$ to $3000 \mathrm{~cm}^{-1}$ as the total concentration of the solution is increased. At low concentrations, we can see two clear peaks at $3414 \mathrm{~cm}^{-1}$ and $3530 \mathrm{~cm}^{-1}$, which are attributed to the in-phase and out-of-phase vibrations of the $\mathrm{NH}_{2}$ group respectively ${ }^{12}$. It should be noted that, even at the lowest concentrations we studied, there is a shoulder on the $3530 \mathrm{~cm}^{-1}$ peak, which we are unable to assign accurately. At larger concentrations, the peaks at $3414 \mathrm{~cm}^{-1}$ and $3530 \mathrm{~cm}^{-1}$ remain, but the shoulder develops and a broad conglomerate of a number of peaks at lower energies (to the left of $3414 \mathrm{~cm}^{-1}$ ) appears. It is well known that hydrogen bonding of the donor $\mathrm{N}-\mathrm{H}$ group leads to a red-shift of the $\mathrm{N}-\mathrm{H}$ vibration frequency from that of the 
free group ${ }^{26}$, so all peaks that appear as the concentration of acrylamide solution increases are assigned to absorption by $\mathrm{NH}_{2}$ groups in different hydrogen-bonded states.

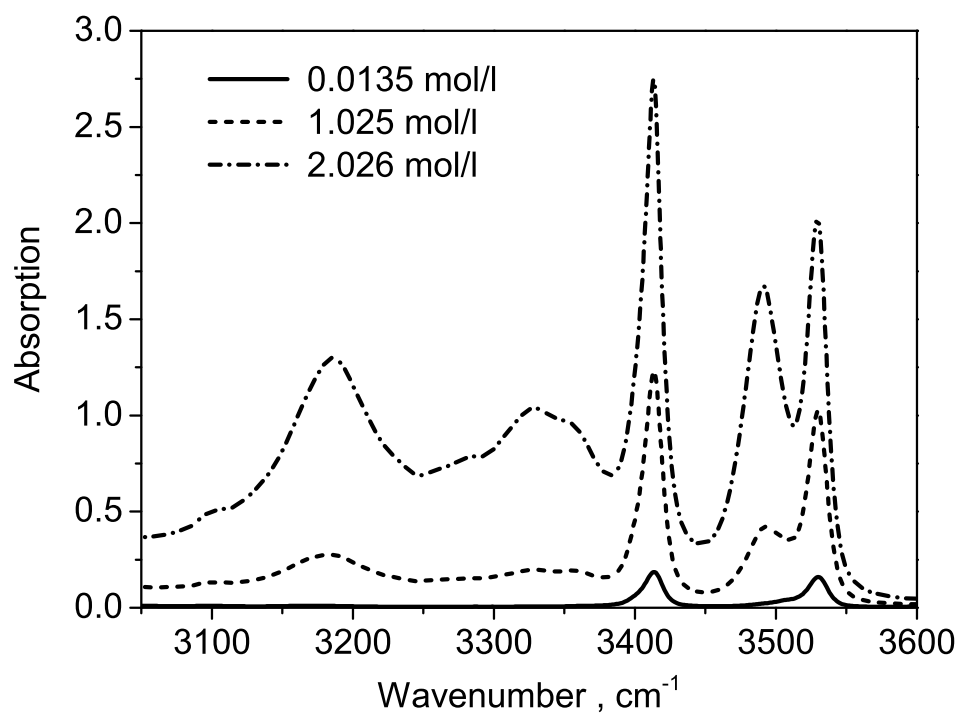

Figure 2: IR spectrum of acrylamide in chloroform at $22^{\circ} \mathrm{C}$ at different concentrations focusing on the $\mathrm{NH}_{2}$ absorption region of spectrum for acrylamide.

However, one must be cautious with respect to the attribution of the $3414 \mathrm{~cm}^{-1}$ and $3530 \mathrm{~cm}^{-1}$ peaks to the "free" $\mathrm{NH}_{2}$ groups, because the bonding state of the oxygen in the same amide group can influence the frequency of absorption. Extensive attempts to rationalize the rules according to which hydrogen bonding affects the absorption wavelengths in amides resulted in a conclusion that there are no universal rules and that each amide system should be carefully studied in order to find the contributions to the shifts in each particular case ${ }^{27-30}$. DFT simulations of hydrogen bonded aggregates of acrylamide could potentially have shed some light on this matter, but we are not aware of any such work in the literature, and the closest study we could find was carried out for perfluorinated polyamides ${ }^{21}$. In their work, the shifts in absorption wavenumbers of the $\mathrm{NH}_{2}$ group in linear and cyclic dimers and trimers were calculated. Interestingly, the shift of the out-ofphase vibrations of the free $\mathrm{NH}_{2}$ groups in the linear dimer was calculated to be $6 \mathrm{~cm}^{-1}$ and in the linear trimer to be $15 \mathrm{~cm}^{-1}$. When applied to the spectra of acrylamide, this 
would result in a contribution of the free groups of linear dimers to the $3530 \mathrm{~cm}^{-1}$ band but would also mean that the absorption of the free group in the trimer would lie away from this band. Similar behavior was reported for acetamide clusters ${ }^{15}$. Consequently, based on the available information, the following possibilities for peak attribution have been considered. The first possibility is that the "free" peaks correspond to unimers. This assumption implies that any bonding of oxygen in the amide group substantially shifts the absorption of the $\mathrm{NH}_{2}$ group. The opposite possibility is that the "free" peaks correspond to the free $\mathrm{NH}_{2}$ groups regardless of the bonding state of oxygen in the same amide group. Finally, the third possibility considered is that these peaks correspond to the absorption of the free $\mathrm{NH}_{2}$ groups in only unimers and dimers, which would correspond to the case when the shift of absorption of a free $\mathrm{NH}_{2}$ group in a dimer is small enough to give a contribution to the $3530 \mathrm{~cm}^{-1}$ peak together with free molecules, but the shift of absorption in free $\mathrm{NH}_{2}$ groups in larger aggregates is large enough not to give a contribution to the $3530 \mathrm{~cm}^{-1}$ peak.

Returning to model 1, one can find now the dependence of the concentration of free molecules and free groups on the total concentration of the solution.

Let us first find the dependence of the total concentration on the concentration of free molecules. Hydrogen bonding is described in the current work as a reversible chemical reaction that produces a range of aggregates of different structures and sizes, and it is assumed that all aggregates are tree-like and no cycles can be formed. In this case, the concentration of aggregates of size $i$ has the form $c_{i}=\alpha_{i} K^{i-1} c_{1}^{i}$ where $K$ is the equilibrium association constant, $c_{1}$ is the concentration of free molecules and $\alpha_{i}$ is a coefficient that depends on the size of the aggregate. With knowledge of $c_{i}$, the total concentration of the solution $n$ and the concentration of bonds $m$ can be calculated to be

$$
n=\sum_{i=1}^{\infty} i c_{i}=c_{1} \sum_{i=1}^{\infty} i \alpha_{i}\left(K c_{1}\right)^{i-1}
$$


and

$$
m=\sum_{i=1}^{\infty}(i-1) c_{i}=c_{1} \sum_{i=1}^{\infty}(i-1) \alpha_{i}\left(K c_{1}\right)^{i-1}
$$

Next, the function

$$
g(x)=\sum_{i=1}^{\infty} \alpha_{i} x^{i}
$$

is introduced, where $x=K c_{1}$; then, $K m=x g^{\prime}-g, K(n-m)=g$ and $K(2 n-m)=x g^{\prime}+g$. Substituting these expressions into Equation 5 yields

$$
x g^{\prime}(x)-g(x)=2 g(x)\left(x g^{\prime}(x)+g(x)\right) .
$$

The solution of this equation that remains finite as $x \rightarrow 0$ is 31

$$
g(x)=\frac{\exp A-4 x-\sqrt{\exp 2 A-8 x \exp A}}{8 x},
$$

where $A$ is a constant determined by the boundary conditions (i.e. the value of $\alpha_{1}$, which is put everywhere equal to 1). We can find $\alpha_{i}$ by expanding Equation 14 as a Taylor series. The general formula for $\alpha_{i}$ is

$$
\alpha_{i}=2^{i-1} \cdot \frac{(2 i) !}{i !(i+1) !}
$$

The sequence $\beta_{i}=\frac{(2 i) !}{i !(i+1) !}$ is known as the Catalan numbers ${ }^{32}$. It is known that the Catalan numbers represent the number of different rooted binary trees with $i+1$ leaves. In the current case, there is an additional factor of $2^{i-1}$, since each molecule apart from the root can be added in two ways to form a bond with one of the free hydrogens as there are two bonding sites on the oxygen. It can then be said that the physical meaning of $\alpha_{i}$ is the number of ways to compose an aggregate of size $i$ out of $i$ molecules. All aggregates allowed in model 1 with size up to $i=3$ are shown in Figure 3.

Substituting the expression for $\alpha_{i}$ into Equation 10, a relation between the concentration 


$$
{ }_{\mathrm{O}}^{\infty} \equiv{ }_{\mathrm{H}^{-}}^{\dot{\mathrm{O}}:}
$$
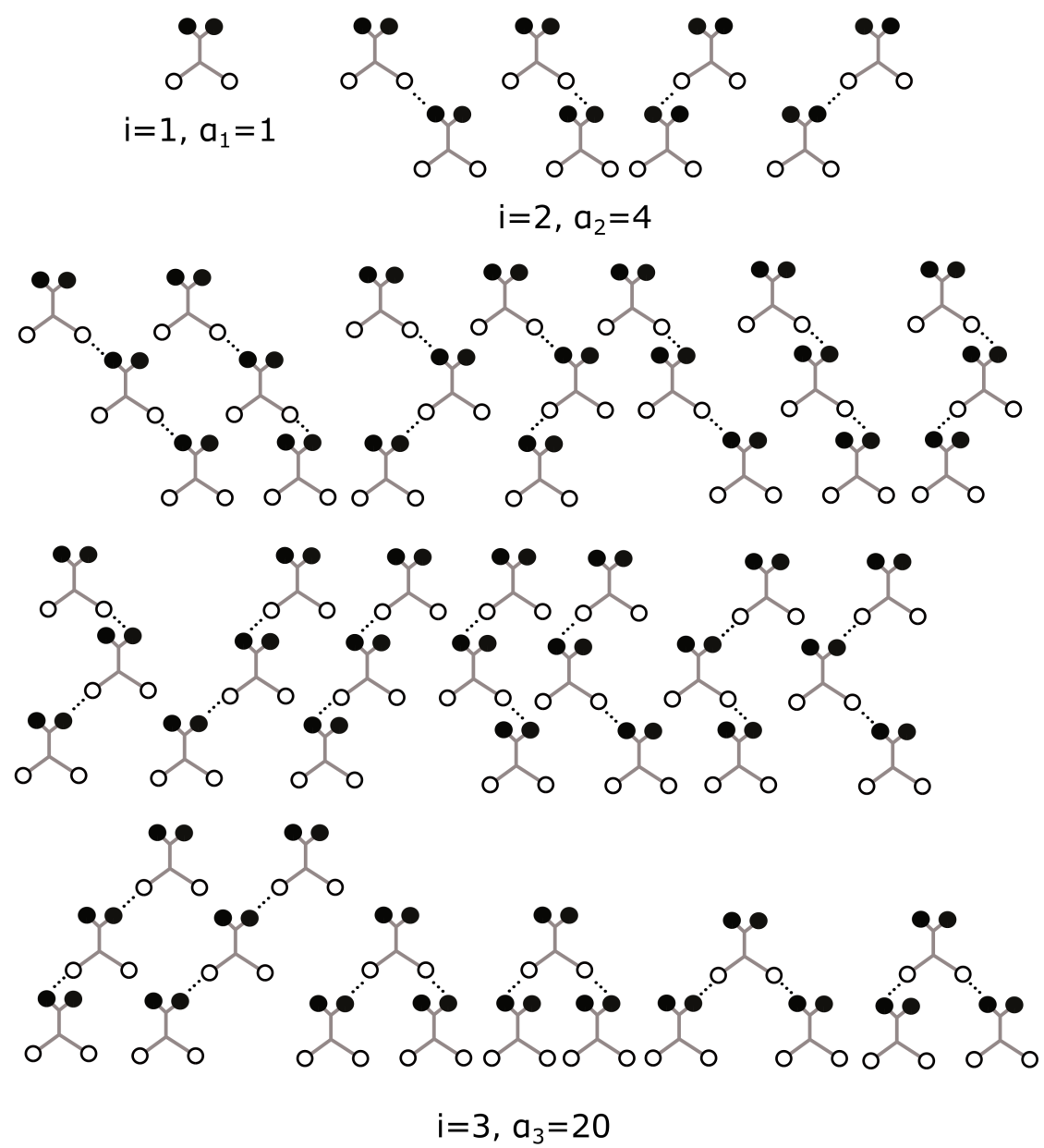

Figure 3: Schematic representation of aggregates with size up to $i=3$ in model 1 . 
of free molecules and the total concentration of the solution is obtained:

$$
n=\frac{1-4 c_{1} K-\sqrt{1-8 c_{1} K}}{8 c_{1} K^{2} \sqrt{1-8 c_{1} K}} .
$$

This expression was used to fit our IR spectroscopy data in the case where the $3530 \mathrm{~cm}^{-1}$ peak is attributed to free molecules, because in this case we simply write $c_{1}=A x$ where $A$ is some constant and $x$ is a peak height.

It is straightforward to obtain from this expression a fitting equation for the case where the peak is attributed to both free molecules and free groups in dimers. In this case $A x=$ $c_{1}+4 K c_{1}^{2}$

Finally, the case where the peak is attributed to free $\mathrm{NH}_{2}$ groups needs to be considered. In order to find the fitting equation, it is necessary to find the concentration of free groups. However, the number of free groups depends on the structure of the aggregate. The most convenient way to do these calculations is to consider the model with two association constants determined by the bonding state of the $\mathrm{NH}_{2}$ group in the donor molecule (see Figure 4) and, after the calculations are complete, put the association constants equal to each other. So, we will assume that the energy of the bond is $\epsilon_{1}$ in the case when a second proton in a donor molecule is free and $\epsilon_{2}$ otherwise. For the sake of clarity, further calculations are omitted here (these can be found in the supporting information in Section 1.6), and the final result for the dependence of the total concentration $n$ on the number of free groups $n_{f}$ is

$$
n=\frac{1-4 K n_{f}+16 K^{2} n_{f}^{2}-\left(1-4 K n_{f}\right) \sqrt{1+16 K^{2} n_{f}^{2}}}{8 K^{2} n_{f}}
$$

For other models all calculations can be found in the Supporting Information.

With all this information in hand, the association constants corresponding to different models can be determined by fitting the dependence of the height of the $3530 \mathrm{~cm}^{-1}$ peak on the total concentration of the solution. In practice, the inverse dependence $n(x)$ will be 

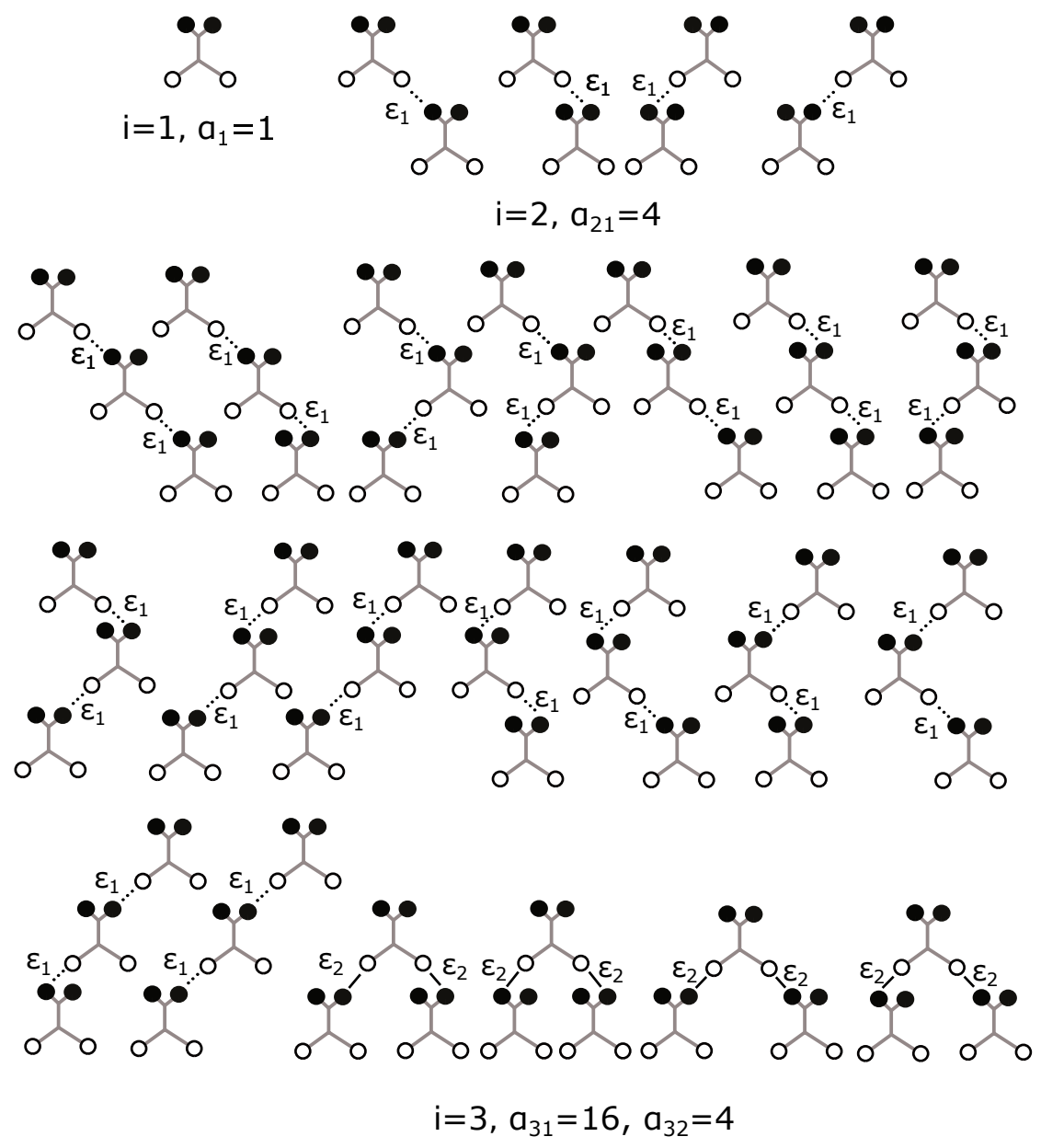

Figure 4: Schematic representation of aggregates with size up to $i=3$ in the extension of model 1 when we assume that the energy of the bond is $\epsilon_{1}$ in the case when a second proton in a donor molecule is free and $\epsilon_{2}$ otherwise. 
fitted for numerical convenience.

The fitting result for model 1 with the free molecules assumption is shown in Figure 5, and the corresponding result with the free groups assumption is shown in Figure 6. The quality of the fit is visibly better with the free molecules assumption, and this point will be discussed in more detail below.

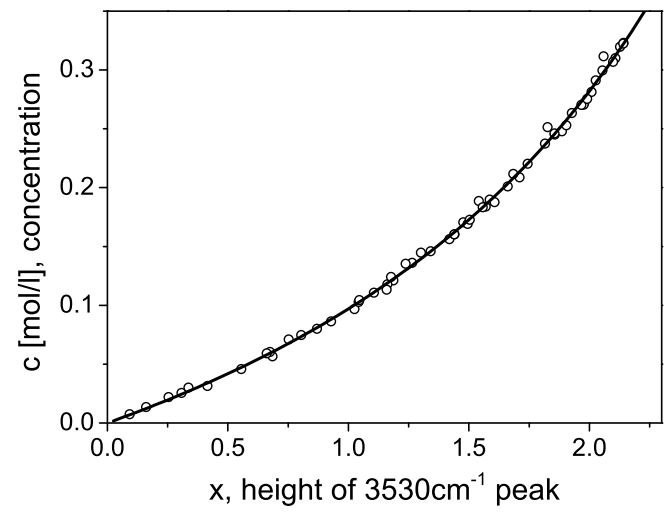

Figure 5: Fitting of the dependence of the total concentration on the height of the $3530 \mathrm{~cm}^{-1}$ IR band at $T=22^{\circ} \mathrm{C}$ with model 1 with the free molecules assumption.

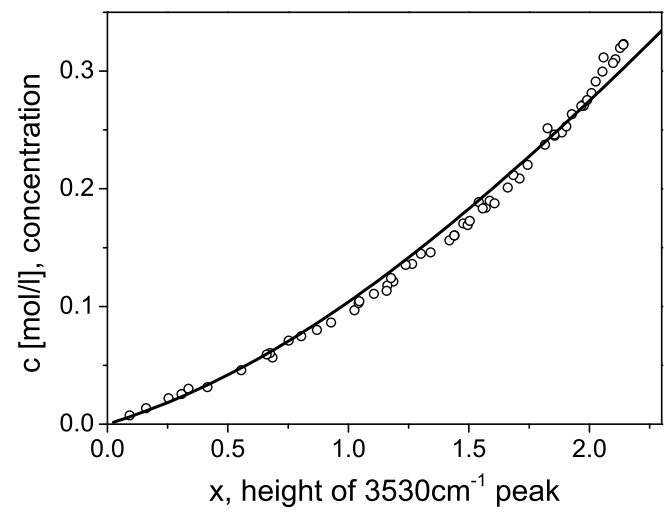

Figure 6: Fitting of the dependence of the total concentration on the height of the $3530 \mathrm{~cm}^{-1}$ IR band at $T=22^{\circ} \mathrm{C}$ with model 1 with free groups assumption.

The results of fitting IR data at $22^{\circ} \mathrm{C}$ are presented in Table 3 . The first two columns specify the number of the model and peak attribution assumption respectively. In the third column, the values of the association constants obtained as fitting parameters are shown. In 
the fourth column, the values of the dimensionless constants are given, which were calculated as $K^{\prime}=K / v$ where $v=0.0629 \mathrm{l} / \mathrm{mol}$ is the molar volume of acrylamide based on its density ${ }^{19}$. The final column gives the values of the AICc parameter ${ }^{33}$, which characterizes the quality of the non-linear fit ${ }^{34,35}$. It can be seen that approximately half of the models give the same quality of fit, so based on fitting results exclusively it cannot be said which model is better. However, attributing the peak to free molecules gives a better quality of fit than attributing it to free groups.

Table 3: IR data fitting results with models with one association constant.

\begin{tabular}{|l|c|c|c|l|}
\hline Model & Peak attr. & $\mathrm{K}$ at $22^{\circ} \mathrm{C},[\mathrm{l} / \mathrm{mol}]$ & $K^{\prime}$ at $22^{\circ} \mathrm{C}$ & AICc at $22^{\circ} \mathrm{C}$ \\
\hline $\mathbf{0}$ & $\mathrm{m}^{a}$ & 2.4 & 38.2 & -457.6 \\
\hline $\mathbf{0}$ & $\mathrm{g}^{b}$ & 1.36 & 21.6 & -298.0 \\
\hline $\mathbf{1}$ & $\mathrm{m}$ & 0.42 & 6.68 & -503.8 \\
\hline $\mathbf{1}$ & $\mathrm{g}$ & 2.37 & 37.68 & -388.9 \\
\hline $\mathbf{1}$ & $\mathrm{s}^{c}$ & 0.65 & 10.30 & -503.9 \\
\hline $\mathbf{2}$ & $\mathrm{m}$ & 0.334 & 5.31 & -502.5 \\
\hline $\mathbf{2}$ & $\mathrm{g}$ & 1.06 & 16.82 & -499.7 \\
\hline $\mathbf{2}$ & $\mathrm{s}$ & 0.442 & 7.03 & -494.2 \\
\hline $\mathbf{3}$ & $\mathrm{m}$ & 0.42 & 6.68 & -499.7 \\
\hline $\mathbf{3}$ & $\mathrm{g}$ & 4.8 & 76.3 & -457.6 \\
\hline $\mathbf{3}$ & $\mathrm{s}$ & 1.02 & 16.22 & -499.3 \\
\hline $\mathbf{4}$ & $\mathrm{m}$ & 0.42 & 6.68 & -503.8 \\
\hline $\mathbf{4}$ & $\mathrm{g}$ & 1.332 & 21.18 & -495.8 \\
\hline $\mathbf{4}$ & $\mathrm{s}$ & 0.65 & 10.30 & -503.9 \\
\hline
\end{tabular}

${ }^{a}$ free molecules assumption ${ }^{b}$ free groups assumption ${ }^{c}$ assumption that $3530 \mathrm{~cm}^{-1}$ peak corresponds to free $\mathrm{NH}_{2}$ groups in unimers and dimers.

Next, the bond energies are determined from the association constants. In order to do this, we perform our measurements and fitting procedures at several different temperatures. Since $\ln K=\ln C-\frac{\epsilon}{k T}$ by definition, $\epsilon$ can be determined from a plot of $\ln K$ against $1 / T$. It is noteworthy that this procedure can serve as an additional test for the model, because if the model fits the data well then $\ln K(1 / T)$ is a linear function with a negative value of the energy of bond formation, $\epsilon<0$. Examples of such plots are shown in Figures 7 and 8 for model 1 with the free molecules and free groups assumptions. The corresponding quantities $C^{\prime}$ and $\epsilon^{\prime}$ for the dimensionless association constant $K^{\prime}$ are shown in Table 4 . The first two 
columns in the table specify the model and peak attribution assumption. The third and fourth columns give the values of $\ln C^{\prime}$ and $\epsilon$; respectively, and the values of the coefficients of determination characterizing the quality of the fit of $\ln K^{\prime}$ vs. $1 / T$ are listed in the last column.

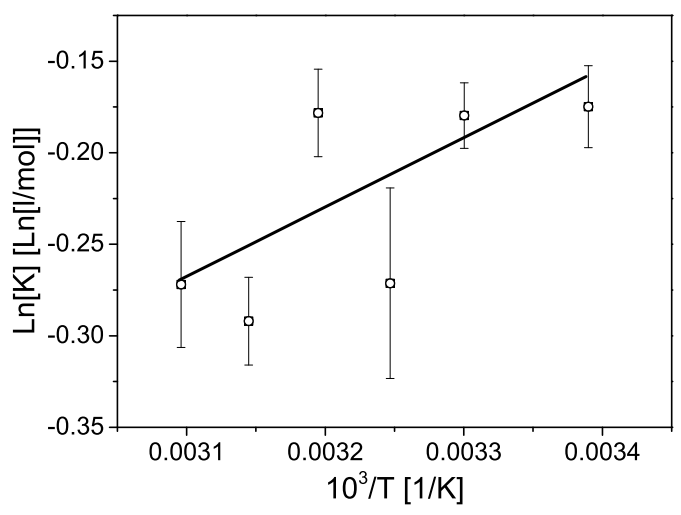

Figure 7: Dependence of $\ln K$ on $1 / T$ for model 1 with the free molecules assumption.

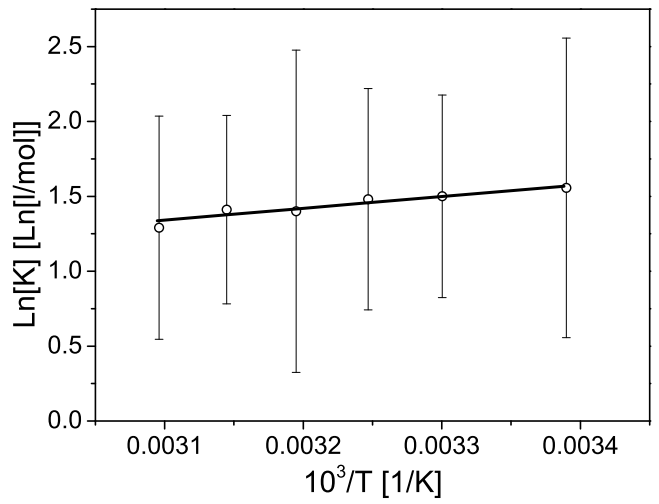

Figure 8: Dependence of $\ln K$ on $1 / T$ for model 1 with the free groups assumption.

It can be seen that the absolute values of the predicted bond energies are close to $1 \mathrm{kcal} / \mathrm{mol}$ for all models. However, according to DFT calculations, the absolute value of the bond energy in an acrylamide dimer in a vacuum can be estimated as $7-9 \mathrm{kcal} / \mathrm{mol}^{12,13}$, which is much larger than the values obtained for one-parameter models.

In addition, one can see that, in the limit of infinite temperature, $T \rightarrow \infty$, we have that $K^{\prime} \rightarrow C^{\prime}$. Since the $C^{\prime}$ values are relatively large (see Table 4 ), $K^{\prime}>1$ for all models 
Table 4: Temperature dependence of (dimensionless) association constants and bond energies for the 'one constant' models.

\begin{tabular}{|l|c|c|c|l|}
\hline Model & Peak attr. & $\ln C^{\prime}$ & $\epsilon,[\mathrm{kcal} / \mathrm{mol}]$ & $r^{2}$ \\
\hline $\mathbf{0}$ & $\mathrm{m}^{a}$ & 1.92 & -1.03 & 0.07 \\
\hline $\mathbf{0}$ & $\mathrm{g}^{b}$ & 1.12 & -1.15 & 0.75 \\
\hline $\mathbf{1}$ & $\mathrm{m}$ & 0.63 & -0.75 & 0.56 \\
\hline $\mathbf{1}$ & $\mathrm{g}$ & 1.00 & -1.55 & 0.9 \\
\hline $\mathbf{1}$ & $\mathrm{s}$ & 1.01 & -0.78 & 0.66 \\
\hline $\mathbf{2}$ & $\mathrm{m}$ & 0.31 & -0.8 & 0.79 \\
\hline $\mathbf{2}$ & $\mathrm{g}$ & 1.61 & -0.72 & 0.38 \\
\hline $\mathbf{2}$ & $\mathrm{s}$ & 0.50 & -0.86 & 0.92 \\
\hline $\mathbf{3}$ & $\mathrm{m}$ & 0.92 & -0.72 & 0.38 \\
\hline $\mathbf{3}$ & $\mathrm{g}$ & 3.31 & -1.03 & 0.07 \\
\hline $\mathbf{3}$ & $\mathrm{s}$ & 1.57 & -0.72 & 0.37 \\
\hline $\mathbf{4}$ & $\mathrm{m}$ & 0.63 & -0.75 & 0.56 \\
\hline $\mathbf{4}$ & $\mathrm{g}$ & 1.92 & -0.675 & 0.28 \\
\hline $\mathbf{4}$ & $\mathrm{s}$ & 1.01 & -0.78 & 0.66 \\
\hline
\end{tabular}

${ }^{a}$ free molecules assumption ${ }^{b}$ free groups assumption ${ }^{c}$ assumption that $3530 \mathrm{~cm}^{-1}$ peak corresponds to free $\mathrm{NH}_{2}$ groups in unimers and dimers.

even at infinite temperatures. This contradicts our intuitive expectation that the strength of hydrogen bonding substantially decreases as the temperature increases. In one of the following sections, models with two association constants are developed, which give a better quality of fit to the data, allowing to obtain a dimer bond energy closer to DFT predictions, and yield physically reasonable asymptotic behavior at high temperatures.

\section{Properties of models with one association constant}

In the previous section, the concentrations of aggregates of all sizes were determined in models with one association constant. This allows one to study some properties of the models, such as the dependence of the total number of bonds and average size of the aggregates on the total concentration of the solution and on the value of the association constant.

First, let us look at the dependence of the ratio of the concentration of bonds to the concentration of molecules $\mathrm{m} / \mathrm{c}$ on the value of the association constant at fixed total con- 
centration $c$, which is shown in Figure 9.

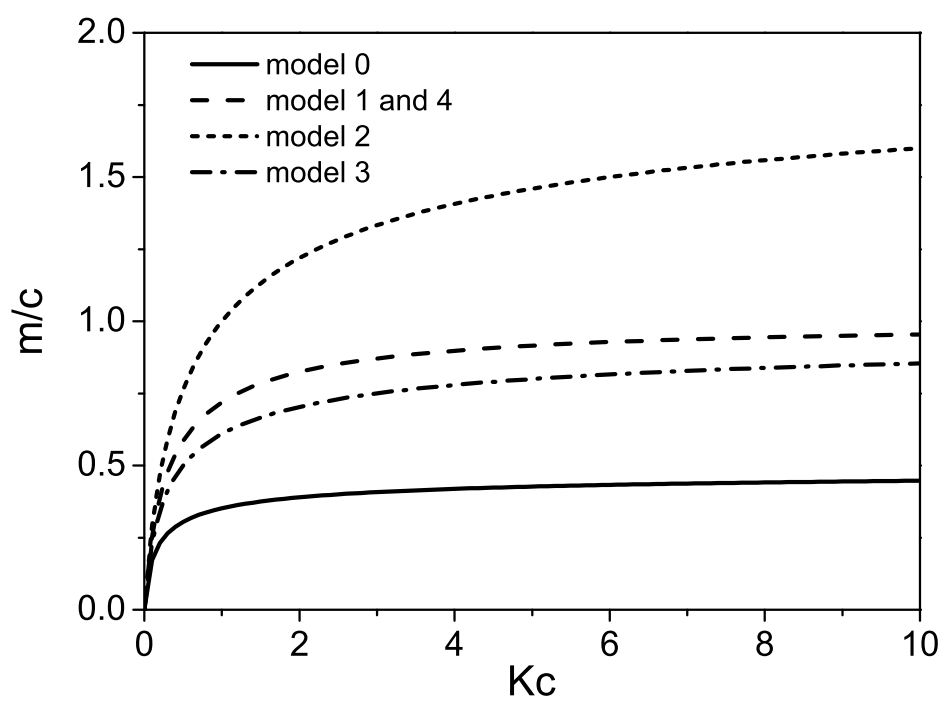

Figure 9: Dependence of $\mathrm{m} / \mathrm{c}$ on $K c$ at fixed total concentration for models with one association constant.

It can be seen that, for model 0 , as $K$ increases, $m / c$ tends to a value of 0.5 in accordance with the assumption that in this model only dimers can form. In the case of models 1,3 , and 4 , the ratio $m / c$ tends to 1 in the limit of infinite $K$. This means that, in these models, the number of bonds is always less then the number of molecules in the system. This is explained by the fact that in all of these models molecules either have one acceptor site or one donor site. In contrast, in model 2 there are two bonding sites of each type for each molecule. As a result, when $K>1 / c$, the number of bonds becomes larger than the number of molecules. In addition, since in an aggregate of size $i$ without cycles the number of bonds is always $i-1$, one can immediately conclude that the assumption about the absence of cycles is wrong when applied to model 2 with $K>1 / c$.

In fact, the restrictions on the applicability of our assumption of the absence of cycles in model 2 are even stronger, because according to our calculations for this model, the total concentration expressed as a function of concentration of unimers can be written in terms of 
a hypergeometric function as

$$
\frac{c}{c_{1}}={ }_{3} F_{2}\left(\left[\frac{4}{3}, \frac{5}{3}, 2\right],\left[\frac{5}{2}, 3\right], \frac{27 K c_{1}}{2}\right) .
$$

The right-hand side of Equation 18 is defined only for $\left|\frac{27 K c_{1}}{2}\right| \leq 1$ and is an increasing function of its argument. The first of these facts means that we must have $c_{1} \leq \frac{2}{27 K}$, and the second means that we must also have $c_{1} \geq c /{ }_{3} F_{2}\left(\left[\frac{4}{3}, \frac{5}{3}, 2\right],\left[\frac{5}{2}, 3\right], 1\right)$. So, when $K>2{ }_{3} F_{2}\left(\left[\frac{4}{3}, \frac{5}{3}, 2\right],\left[\frac{5}{2}, 3\right], 1\right) / 27 c=3 / 8 c$, Equation 18 has no solutions. The ratio of $m$ and $c$ at the maximum value of $K$ is $m / c=2 / 3$, so the assumption about the absence of cycles fails when the number of bonds per molecule becomes larger than $2 / 3$.

Another interesting property is the dependence of the average aggregate size on the values of the association constant and concentration. The average aggregation number can be calculated as

$$
\langle i\rangle=\frac{\sum_{i=1}^{\infty} i c_{i}}{\sum_{i=1}^{\infty} c_{i}}
$$

In Figure 10, the dependence of the average aggregation number on the value of the dimensionless association constant with the volume fraction of acrylamide fixed to the largest experimental volume fraction, $\phi=0.022$, is shown for models $0,1,3$ and 4 . Figure 11 shows the dependence of the average aggregation number on the volume fraction of acrylamide at association constants obtained from IR measurements for models $0 \mathrm{~m}, 1 \mathrm{~m}, 3 \mathrm{~m}$, and $4 \mathrm{~g}$. For all models, $\langle i\rangle$ monotonically increases as $K^{\prime}$ and $\phi$ increase, and, as expected, $\langle i\rangle$ grows more quickly for models 1 and 4 than for model 3 .

In the case of model 2 , at a fixed value of the concentration there is a maximum value of the association constant above which our assumption about the absence of cycles does not work. Correspondingly, for each value of the association constant, there is a maximum concentration of acrylamide above which model 2 again is not applicable. Within the regime where the model is valid, the average aggregation number is an increasing function of concentration and the association constant and reaches its maximum value of 3 when $K c_{1}=2 / 27$. 


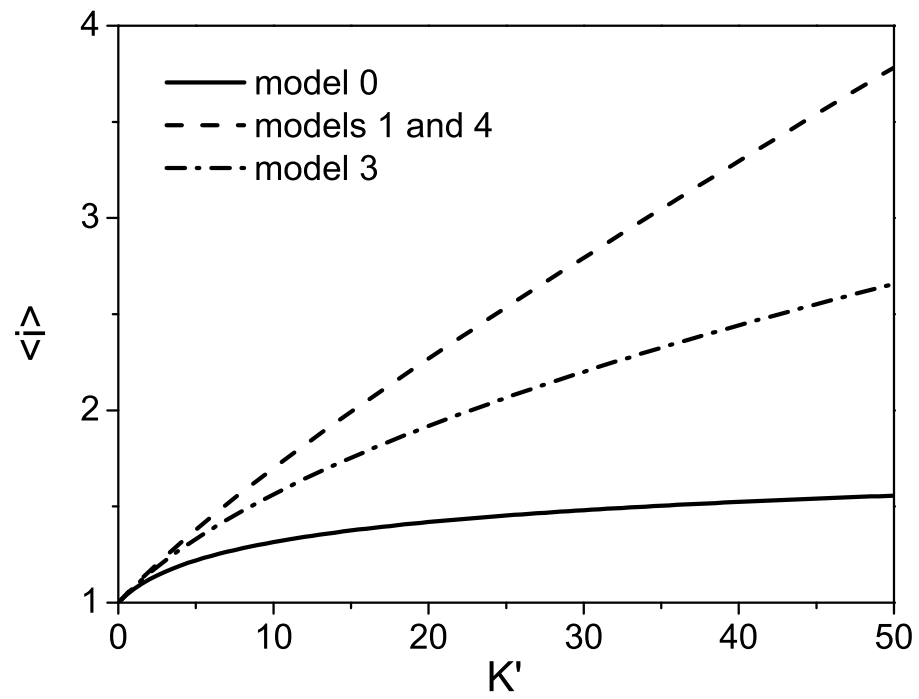

Figure 10: Dependence of $\langle i\rangle$ on the value of the dimensionless association constant at volume fraction of acrylamide fixed to the largest experimental volume fraction, $\phi=0.022$.

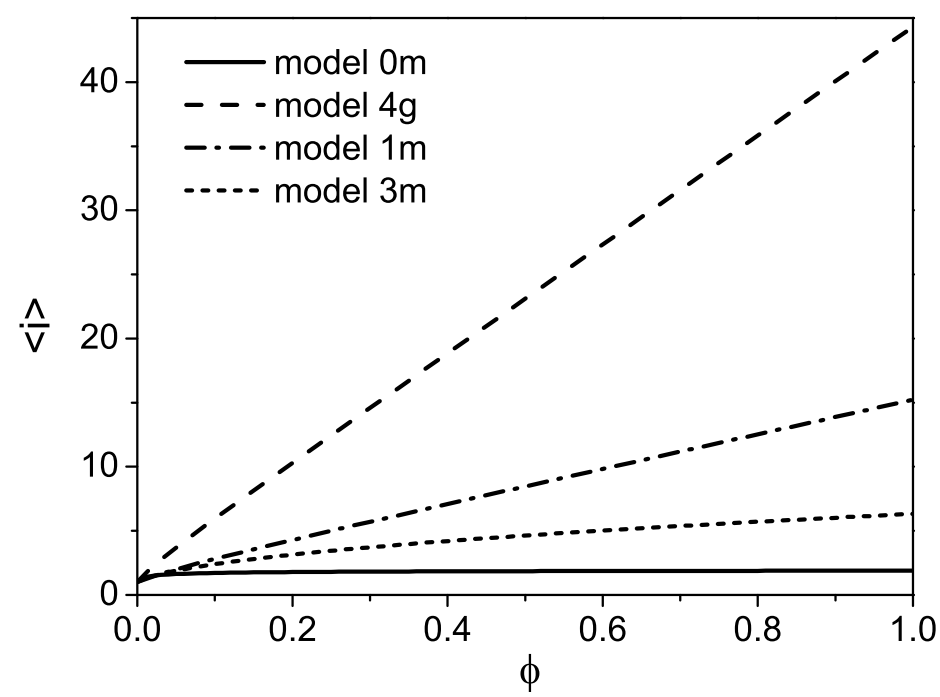

Figure 11: Dependence of $\langle i\rangle$ on the volume fraction of acrylamide with association constant determined from fitting IR data at $T=22^{\circ} \mathrm{C}$. 
This behavior is illustrated in Figure 12, which shows the dependence of the average aggregation number in model 2 on the volume fraction of acrylamide at a fixed value of the association constant.

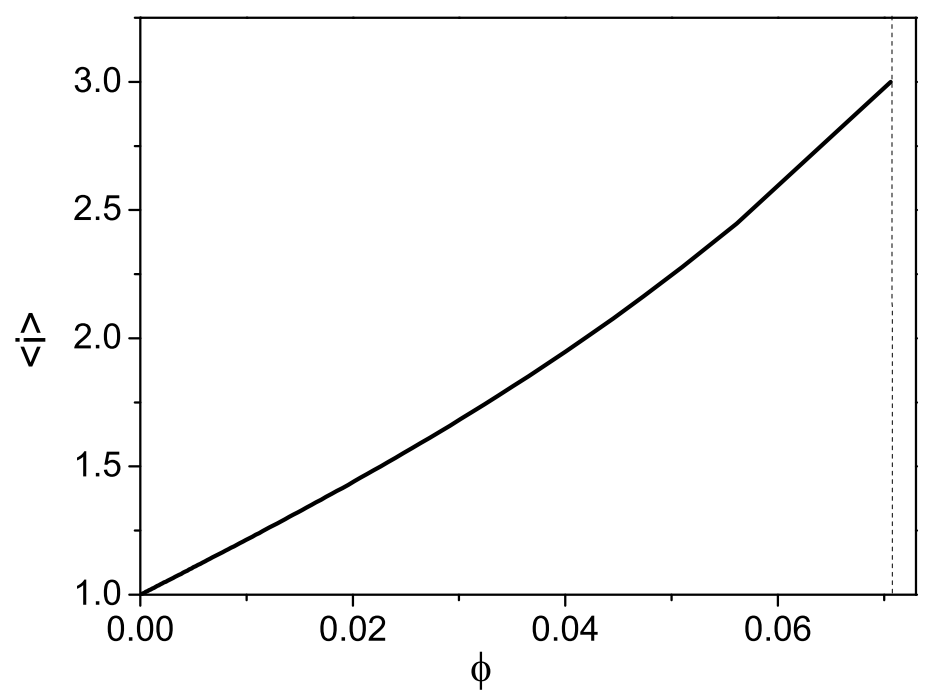

Figure 12: Dependence of $\langle i\rangle$ on volume fraction of acrylamide with association constant determined from fit of IR data at $T=22^{\circ} \mathrm{C}$ for model $2 \mathrm{~m}$.

\section{Models with two association constants}

In this section, we consider models in which association is characterized by two association constants. As in the case of alcohols, these constants depend on the bonding state of the other groups belonging to the molecule forming a given hydrogen bond. Again, it is assumed that cycles cannot form. Here only two-constant extensions of models 1, 3 and 4 are constructed. Model 2 has not been considered here, as this case requires significant additional study ${ }^{36}$.

The list of models with two association constants is presented in Table 5 and the rules for how the energy of a bond depends on its location are illustrated in Figure 13.

All of these cases can be treated analytically and calculations can be found in the Supporting Information. Here, just tables (see Tables 6 and 7) of the values of the association 

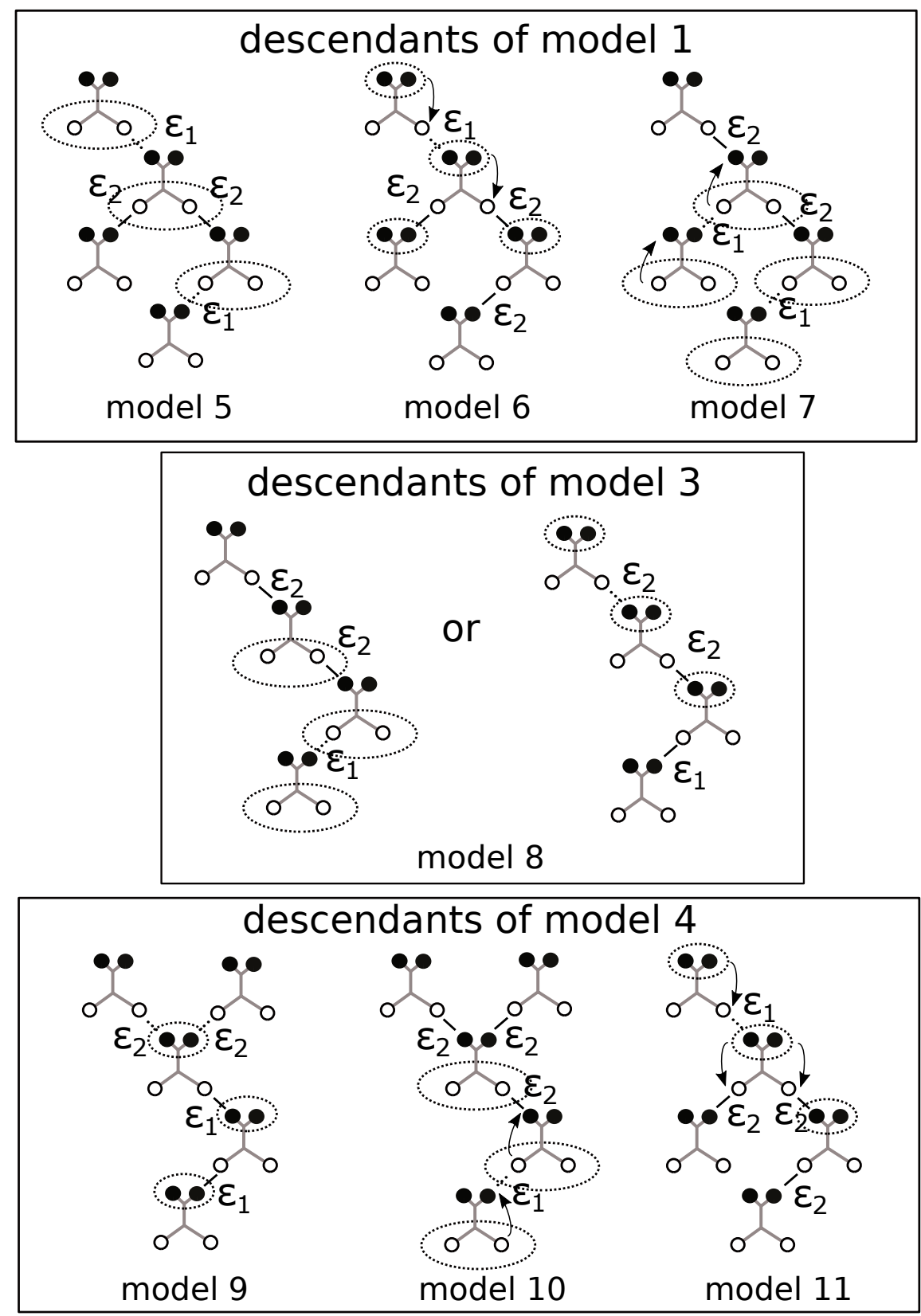

Figure 13: Two-parameter models. 
Table 5: List of models with two association constants and no cycles.

\begin{tabular}{|l|l|}
\hline Model & Association rules \\
\hline \hline $\mathbf{5}$ & $\begin{array}{l}\text { one bond per oxygen, two bonds per } \mathrm{NH}_{2} \text { group, } \\
\text { energy of hydrogen bond is determined by the bonding state } \\
\text { of the neighbor hydrogen in } \mathrm{NH}_{2} \text { group of the donor molecule }\end{array}$ \\
\hline $\mathbf{6}$ & $\begin{array}{l}\text { one bond per oxygen, two bonds per } \mathrm{NH}_{2} \text { group, } \\
\text { energy of hydrogen bond is determined by the bonding state } \\
\text { of acceptor in donor molecule }\end{array}$ \\
\hline $\mathbf{7}$ & $\begin{array}{l}\text { one bond per oxygen, two bonds per } \mathrm{NH}_{2} \text { group, } \\
\text { energy of hydrogen bond is determined by the bonding state } \\
\text { of the NH}\end{array}$ \\
\hline $\mathbf{8}$ & $\begin{array}{l}\text { one bond per oxygen, one bond per } \mathrm{NH}_{2} \text { group, } \\
\text { bond energy is determined by bonding state of acceptor in donor molecule } \\
\text { or bond energy is determined by bonding state of donor in acceptor molecule } \\
\text { (both definitions give equivalent results) }\end{array}$ \\
\hline $\mathbf{9}$ & $\begin{array}{l}\text { two bonds per oxygen, one bond per } \mathrm{NH}_{2} \text { group, } \\
\text { bond energy depends on bonding state of acceptor in acceptor molecule }\end{array}$ \\
\hline $\mathbf{1 0}$ & $\begin{array}{l}\text { two bonds per oxygen, one bond per } \mathrm{NH}_{2} \text { group, } \\
\text { bond energy depends on bonding state of } \mathrm{NH}_{2} \text { group in acceptor molecule }\end{array}$ \\
\hline $\mathbf{1 1}$ & $\begin{array}{l}\text { two bonds per oxygen, one bond per } \mathrm{NH}_{2} \text { group, } \\
\text { bond energy depends on bonding state of oxygen group in donor molecule }\end{array}$ \\
\hline
\end{tabular}

constants for these models are shown.

The first thing to note is that the quality of fitting increases as we turn to models with two association constants. Based on the combination of $\mathrm{AICc}$ and $r_{i}^{2}$ for the fits of $c(x)$ and $\ln K_{i}(1 / T)$, we can conclude that models $6 \mathrm{~s}$ (and the equivalent model 10s), 8s and $10 \mathrm{~g}$ are good. All of these models give the value of the bond energy in a dimer as about $6-8 \mathrm{kcal} / \mathrm{mol}$, which corresponds much more closely to the values calculated by $\mathrm{DFT}^{12,13}$ discussed earlier. Another important property of these models is that the values they give for both $C_{1}$ and $C_{2}$ are smaller than those found for one-constant models. So, in the limit of infinite temperature, the association constants $K_{1}$ and $K_{2}$ will have smaller values.

Fitting also shows that model 5, which includes the assumption that bonding energy is fixed by the bonding state of the neighbor hydrogen in the $\mathrm{NH}_{2}$ group, is poor. It means that either the bonding state of one hydrogen does not affect the bonding of its neighbor hydrogen (implying that model 5 reduces to model 1) or that the bonding of one of the hydrogens in 
Table 6: IR data fitting results with models with two association constant at $22^{\circ} \mathbf{C}$.

\begin{tabular}{|c|c|c|c|c|c|c|}
\hline Model & Peak attr. & $K_{1},[\mathrm{l} / \mathrm{mol}]$ & $K_{1}^{\prime}$ & $K_{2},[\mathrm{l} / \mathrm{mol}]$ & $K_{2}^{\prime}$ & $\mathrm{AICc}$ \\
\hline 5 & $\mathrm{~m}$ & 0.38 & 6.04 & 0.49 & 7.79 & -501.7 \\
\hline 5 & $\mathrm{~g}$ & \multicolumn{5}{|c|}{ no good convergence } \\
\hline 5 & $\mathrm{~S}$ & 0.69 & 11.0 & 0.61 & 9.7 & -501.7 \\
\hline 6 & $\mathrm{~m}$ & 0.38 & 6.09 & 0.44 & 7.0 & -501.7 \\
\hline 6 & $\mathrm{~g}$ & 0.27 & 4.29 & 1.05 & 16.69 & -493.9 \\
\hline 6 & $\mathrm{~s}$ & 0.72 & 11.45 & 0.69 & 10.92 & -501.7 \\
\hline 7 & $\mathrm{~m}$ & 0.38 & 6.04 & 0.45 & 7.15 & -501.7 \\
\hline 7 & $\mathrm{~g}$ & 0.29 & 4.54 & 1.24 & 19.7 & -497.4 \\
\hline 7 & $\mathrm{~s}$ & 0.74 & 11.8 & 0.68 & 10.8 & -501.7 \\
\hline 8 & $\mathrm{~m}$ & 0.35 & 5.56 & 0.61 & 9.7 & -501.5 \\
\hline 8 & $\mathrm{~g}$ & 0.33 & 5.24 & 1.36 & 21.54 & -499.5 \\
\hline 8 & $\mathrm{~s}$ & 0.56 & 8.9 & 0.9 & 14.3 & -501.4 \\
\hline 9 & $\mathrm{~m}$ & 0.38 & 6.07 & 0.49 & 7.73 & -501.7 \\
\hline 9 & $\mathrm{~g}$ & 0.51 & 8.11 & 1.38 & 21.94 & -501.3 \\
\hline 9 & $\mathrm{~s}$ & 0.70 & 11.05 & 0.61 & 9.70 & -501.7 \\
\hline 10 & $\mathrm{~m}$ & 0.38 & 6.09 & 0.44 & 6.93 & -501.7 \\
\hline 10 & $\mathrm{~g}$ & 0.67 & 10.59 & 1.01 & 16.06 & -501.1 \\
\hline 10 & $\mathrm{~s}$ & 0.72 & 11.49 & 0.69 & 10.92 & -501.7 \\
\hline 11 & $\mathrm{~m}$ & 0.38 & 6.04 & 0.45 & 7.09 & -501.7 \\
\hline 11 & $\mathrm{~g}$ & \multicolumn{5}{|c|}{ no good convergence } \\
\hline 11 & $\mathrm{~S}$ & 0.74 & 11.7 & 0.68 & 10.81 & -501.7 \\
\hline
\end{tabular}


Table 7: Temperature dependence of (dimensionless) association constants and bond energies for models with two association constants.

\begin{tabular}{|l|c|c|c|c|c|c|c|}
\hline Model & Peak attr. & $\ln C_{1}^{\prime}$ & $\epsilon_{1},[\mathrm{kcal} / \mathrm{mol}]$ & $r_{1}^{2}$ & $\ln C_{2}^{\prime}$ & $\epsilon_{2},[\mathrm{kcal} / \mathrm{mol}]$ & $r_{2}^{2}$ \\
\hline $\mathbf{5}$ & $\mathrm{m}$ & -16.8 & -11 & 0.76 & 6.2 & 2.4 & 0.73 \\
\hline $\mathbf{5}$ & $\mathrm{g}$ & \multicolumn{7}{|c|}{ no good convergence } \\
\hline $\mathbf{5}$ & $\mathrm{s}$ & -18.7 & -12.5 & 0.76 & 4.7 & 1.3 & 0.56 \\
\hline $\mathbf{6}$ & $\mathrm{m}$ & -7.2 & -5.2 & 0.91 & 2.2 & 0.1 & 0.03 \\
\hline $\mathbf{6}$ & $\mathrm{g}$ & -8.7 & -5.9 & 0.71 & -1.0 & -2.2 & 0.90 \\
\hline $\mathbf{6}$ & $\mathrm{s}$ & -9.1 & -6.7 & 0.91 & -0.4 & -1.6 & 0.95 \\
\hline $\mathbf{7}$ & $\mathrm{m}$ & -8.9 & -6.2 & 0.9 & 4.1 & 1.2 & 0.62 \\
\hline $\mathbf{7}$ & $\mathrm{g}$ & -9.6 & -6.5 & 0.8 & -0.13 & -1.8 & 0.95 \\
\hline $\mathbf{7}$ & $\mathrm{s}$ & -11.6 & -8.2 & 0.9 & 1.29 & -0.6 & 0.7 \\
\hline $\mathbf{8}$ & $\mathrm{m}$ & -9.5 & -6.6 & 0.89 & 2.85 & 0.3 & 0.18 \\
\hline $\mathbf{8}$ & $\mathrm{g}$ & -10.8 & -7.3 & 0.83 & -0.39 & -2 & 0.95 \\
\hline $\mathbf{8}$ & $\mathrm{s}$ & -11.4 & -7.9 & 0.88 & 0.37 & -1.3 & 0.93 \\
\hline $\mathbf{9}$ & $\mathrm{m}$ & -16.8 & -11 & 0.76 & 6.2 & 2.4 & 0.73 \\
\hline $\mathbf{9}$ & $\mathrm{g}$ & $\mathrm{n}$ & good convergence at higher temperatures & \\
\hline $\mathbf{9}$ & $\mathrm{s}$ & -18.7 & -12.5 & 0.76 & 4.7 & 1.3 & 0.56 \\
\hline $\mathbf{1 0}$ & $\mathrm{m}$ & -7.2 & -5.2 & 0.91 & 2.2 & 0.1 & 0.03 \\
\hline $\mathbf{1 0}$ & $\mathrm{g}$ & -8.5 & -6.3 & 0.9 & -1.41 & -2.4 & 0.95 \\
\hline $\mathbf{1 0}$ & $\mathrm{s}$ & -9.1 & -6.7 & 0.91 & -0.4 & -1.6 & 0.95 \\
\hline $\mathbf{1 1}$ & $\mathrm{m}$ & -8.9 & -6.2 & 0.9 & 4.1 & 1.2 & 0.61 \\
\hline $\mathbf{1 1}$ & $\mathrm{g}$ & $\mathrm{g}$ & \multicolumn{7}{|c|}{ no good convergence } & \\
\hline $\mathbf{1 1}$ & $\mathrm{s}$ & -11.6 & -8.2 & 0.9 & 1.29 & -0.6 & 0.69 \\
\hline
\end{tabular}


the $\mathrm{NH}_{2}$ group leads to the complete loss of the donor properties of the second hydrogen (so that model 5 reduces to model 3). The same observation applies to the hydrogen bonding sites on oxygen in model 9 which reduces either to model 4 or to model 3.

Similar conclusions can be made with regard to the pair of models 7 and 11, because they show worse fitting results than models 6,8 and 10. As a result, it can be concluded that the assumption that there are only one or two bonds with energy $\epsilon_{1}$ at the "top" of each aggregate and all other bonds have energy $\epsilon_{2}$ is the most probable one according to the fitting results.

It is interesting to note that, in all cases, the value of $\epsilon_{2}$ is smaller than the value of $\epsilon_{1}$, so the formation of the initial dimer is a more energetically favorable process than the addition of subsequent acrylamide molecules to the aggregate. It is also interesting to note that this difference is much larger than in the similar situations in alcohols ${ }^{3}$.

We also can see that there is a contradiction with DFT calculations on formamide and acetamide linear clusters, which predict that the energy per bond increases with the growth of the size of the linear cluster ${ }^{15,37}$. However, as both approaches involve their own approximations, additional study is needed to understand the reasons for this discrepancy.

\section{Structure factor of diblock copolymer with hydrogen bond- ing block in a disordered state}

Hydrogen-bonding block copolymers are exploited in various areas of polymer science ${ }^{38-40}$. For example, it was recently proposed to use block copolymers with one hydrogen-bonding block and one non-hydrogen-bonding block for lithographic applications since these block copolymer systems possess an enhanced tendency to separation ${ }^{41,42}$. The physical reason for this is the fact that in order to mix a non-hydrogen-bonding block and a hydrogenbonding block one needs to break the network of hydrogen bonds, which is energetically expensive. Usually, interactions in such systems are characterized by a single Flory-Huggins 
parameter ${ }^{6-8,41,42}$, which is determined by means of fitting the scattering function of a diblock copolymer melt in a disordered state with the theoretical expression depending on $\chi$. Here, we add a short discussion of a scattering function calculation for a diblock copolymer with one hydrogen-bonding and one non-hydrogen-bonding block in a random mixing approximation as a starting point for further developments and as a simple illustration of the fact that the models of hydrogen bonding that do not take into account the non-randomness of the number of contacts between hydrogen bonding segments are inadequate.

According to one of the basic assumptions of the association model approach, the hydrogenbonding contribution can be isolated from all other contributions to the free energy, so we have $F=F_{0}+F_{\mathrm{HB}}$ where $F_{0}$ is the free energy of the system (of total volume $V$ ) without hydrogen bonds and $F_{\mathrm{HB}}$ is the contribution due to hydrogen bonding. Then, the scattering function in the disordered state in the $\mathrm{RPA}^{5,43}$, or in other words in the random mixing approximation, takes the form

$$
\begin{gathered}
S^{-1}(k)=S_{0}^{-1}(k)+\frac{d^{2} f_{\mathrm{HB}}(f)}{d \phi_{B}^{2}}=\frac{g(x, 1)}{N \operatorname{det}(S)}-2\left(\chi-\frac{1}{2} \frac{d^{2} f_{\mathrm{HB}}(f)}{d \phi_{B}^{2}}\right) \\
\chi_{\mathrm{eff}}=\chi-\frac{1}{2} \frac{d^{2} f_{\mathrm{HB}}(f)}{d \phi_{B}^{2}}
\end{gathered}
$$

where ${ }^{5} S_{0}^{-1}(k)$ is the inverse scattering function of the block copolymer without hydrogen bonds and $\frac{d^{2} f_{\mathrm{HB}}(f)}{d \phi_{B}^{2}}$ is always negative. So, one can see that in the system with a hydrogenbonding block the value of $\chi_{\text {eff }}$ determined by means of fitting a scattering function with the regular expression $S_{0}^{-1}(k)$ will consist of two contributions, the first one, $\chi$, describing nonspecific interactions, and the second one, $\delta \chi_{\mathrm{HB}}(f)$, describing the contribution of hydrogen bonds and depending on the volume fraction of the hydrogen-bonding block. This also means that the value of the Flory-Huggins parameter, $\chi_{\mathrm{eff}}$, determined in scattering experiments cannot be used to describe interactions in diblock copolymers with hydrogen bonds in the form $\chi_{\mathrm{eff}} \phi(1-\phi)$ and the initial expression $F / k T V=\chi \phi(1-\phi)+f_{\mathrm{HB}}(\phi)$ should be used instead. 
In general, $\delta \chi_{\mathrm{HB}}(f)=-\frac{1}{2} \frac{d^{2} f_{\mathrm{HB}}(f)}{d \phi_{B}^{2}}$ can be determined separately if the association constants for the hydrogen-bonding block are known ${ }^{44}$. Calculations of $\delta \chi_{\mathrm{HB}}$ in the random mixing approximation for acrylamide can be found in the Supporting Information.

The assumption that mixing is, in general, random for polymer systems is not true and several ideas have been proposed to address it in different areas of polymer theory ${ }^{45-47}$. Painter, Veytsman and Coleman also proposed an approach to this problem for mixtures of hydrogen-bonding homopolymers ${ }^{47}$. For hydrogen-bonding block copolymers, the nonrandomness of mixing also must be taken into account, however here we have considered only the random mixing approximation as a starting point for further discussion.

\section{Conclusion}

In this work, an extension of the association model approach was developed in order to describe the association of molecules with two hydrogen acceptor and two hydrogen donor sites. Models with one association constant were considered, in which it is supposed that all bonds have the same energy, and models with two association constants, in which the bond energy is determined by the local hydrogen-bonding environment.

These models are used to fit FTIR experimental data on solutions of acrylamide in chloroform in order to determine the association constants and their temperature dependence for acrylamide, and it was found that several models give the same quality of fitting of experimental data. However, models with two association constants in general give better fits than models with one association constant. Moreover, the bond energies in hydrogen bonding dimers for two-constant models are close to the predictions of DFT calculations, which is not the case in one-constant models.

It was also found that, in systems in which two bonds per acceptor site and two bonds

per donor site are allowed (such as water), the assumption that cycles are absent ceases to be valid at small concentrations of the hydrogen-bonding substance and there is no non- 
cyclic solution of the model when the ratio between the number of bonds and number of molecules is larger than $2 / 3$. Interestingly, the largest average aggregation number possible in this model is equal to 3 . Based on this result, one can conclude that in such substances as acrylamide taking into account formation of cycles is essential.

Finally, the structure factor of a disordered state of diblock copolymer with one hydrogenbonding block and one non-hydrogen-bonding block was calculated in the random phase

approximation. We showed that the presence of hydrogen bonds shifts the value of the Flory-Huggins parameter that appears in the expression for the inverse scattering function, and that this shifted value depends on the volume fraction of the hydrogen bonding block, $\chi_{\mathrm{eff}}=\chi+\delta \chi_{\mathrm{HB}}(f)$. It also implies that a $\chi$ parameter determined solely from scattering experiments cannot be used to describe interactions in hydrogen-bonding systems.

\section{Experimental}

The FTIR experiments were conducted on a Frontier Perkin Elmer spectrometer. A Specac heatable sealed liquid cell with path length $1 \mathrm{~mm}$ and $\mathrm{NaCl}$ windows was used. Acrylamide $(\geq 99 \%)$ and chloroform (anhydrous, stabilized by amylenes, $\geq 99.8 \%$ ) were purchased from Sigma Aldrich.

\section{Acknowledgement}

E.P. is grateful to the European Research Council for the support of the work by the Marie Sklodowska-Curie IF "HYBOCOMIX" (ID 704459). The authors thank John Lane for discussions of the model selection criteria, Tom McLeish for discussions of the physics of the association model approach, and Simon Smith, Ivan Ado, Noam Zeilberger and Mark van Hoeij for discussions of the graph theoretical part of the work. The authors also thank Mark van Hoeij for the proof that the solution we found for model 2 is a unique one. 


\section{Supporting Information}

Supporting Information includes descriptions and calculations for all models, short reference about AICc criteria for model selection and calculations of $\delta \chi(f)$ for polyacrylamide block in random phase approximation.

\section{References}

(1) Mahadevi, A. S.; Sastry, G. N. Cooperativity in Noncovalent Interactions. Chem. Rev. 2016, 116, 2775-2825.

(2) Vasiltsova, T.; Heintz, A. New statistical mechanical model for calculating Kirkwood factors in self-associating liquid systems and its application to mixtures New statistical mechanical model for calculating Kirkwood factors in self-associating liquid systems and its application to alkanol+ cyclohexane mixtures. J. Chem. Phys 2007, 127, 114501.

(3) Coleman, M. M.; Painter, P. C. Hydrogen-Bonded Polymer Blends. Prog. Polym. Sci. 1995, 20, 1-59.

(4) Kuo, S. W. Hydrogen-bonding in polymer blends. J. Polym. Res. 2008, 15, 459-486.

(5) Matsen, M. W. Soft Matter; Wiley-Blackwell, 2006; Chapter 2, pp 87-178.

(6) Dehghan, A.; Shi, A. C. Modeling hydrogen bonding in diblock copolymer/homopolymer blends. Macromolecules 2013, 46, 5796-5805.

(7) Han, S. H.; Kim, J. K.; Pryamitsyn, V.; Ganesan, V. Phase Behavior of Binary Blends of Block Copolymers Having Hydrogen Bonding. Macromolecules 2011, 44, 4970-4976.

(8) Sunday, D. F.; Hannon, A. F.; Tein, S.; Kline, R. J. Thermodynamic and Morphological Behavior of Block Copolymer Blends with Thermal Polymer Additives. Macromolecules 2016, 49, 4898-4908. 
(9) For two cases of alternative approaches see ${ }^{48,49}$.

(10) Liao, W.-C.; Lilienthal, S.; Kahn, J. S.; Riutin, M.; Sohn, Y. S.; Nechushtai, R.; Willner, I. pH- and ligand-induced release of loads from DNA-acrylamide hydrogel microcapsules. Chem. Sci. 2017, 8, 3362-3373.

(11) Sun, J. Y.; Zhao, X.; Illeperuma, W. R.; Chaudhuri, O.; Oh, K. H.; Mooney, D. J.; Vlassak, J. J.; Suo, Z. Highly stretchable and tough hydrogels. Nature 2012, 489, 133-136.

(12) Duarte, A. S.; Amorim Da Costa, A. M.; Amado, A. M. On the conformation of neat acrylamide dimers - A study by ab initio calculations and vibrational spectroscopy. THEOCHEM 2005, 723, 63-68.

(13) Wang, Y.-S.; Lin, Y.-D.; Chao, S. D. Hydrogen-bonding Structures and Energetics of Acrylamide Isomers, Tautomers, and Dimers: An ab initio Study and Spectral Analysis. J. Chin. Chem. Soc. 2016, 63, 968-976.

(14) Bakó, I.; Megyes, T.; Bálint, S.; Chihaia, V.; Bellissent-Funel, M.-C.; Krienke, H.; Kopf, A.; Suh, S.-H. Hydrogen bonded network properties in liquid formamide. J. Chem. Phys. 2010, 132, 014506.

(15) Esrafili, M. D.; Behzadi, H.; Hadipour, N. L. Theoretical study of N-H . O hydrogen bonding properties and cooperativity effects in linear acetamide clusters. Theor. Chem. Acc. 2008, 121, 135-146.

(16) Mahadevi, A. S.; Neela, Y. I.; Sastry, G. N. A theoretical study on structural, spectroscopic and energetic properties of acetamide clusters [CH3CONH2] $(\mathrm{n}=1-15)$. Phys. Chem. Chem. Phys. 2011, 13, 15211-15220.

(17) Stubbs, J. M.; Siepmann, J. I. Elucidating the vibrational spectra of hydrogen-bonded aggregates in solution: Electronic structure calculations with implicit solvent and first- 
principles molecular dynamics simulations with explicit solvent for 1-hexanol in nhexane. J. Am. Chem. Soc. 2005, 127, 4722-4729.

(18) Spencer, J. N.; Garrett, R. C.; Mayer, F. J.; Merkle, J. E.; Powell, C. R.; Tran, M. T.; Berger, S. K. Amide hydrogen bonding in organic medium. Can. J. Chem. 1980, 58, $1372-1375$.

(19) Udovenko, A. A.; Kolzunova, L. G. Crystal structure of acrylamide. J. Struct. Chem. 2008, 49, 961-964.

(20) Böhmer, R.; Gainaru, C.; Richert, R. Structure and dynamics of monohydroxy alcoholsMilestones towards their microscopic understanding, 100 years after Debye. Phys. Rep 2014, 545, 125-195.

(21) Milani, A.; Castiglioni, C.; Di Dedda, E.; Radice, S.; Canil, G.; Di Meo, A.; Picozzi, R.; Tonelli, C. Hydrogen bonding effects in perfluorinated polyamides: An investigation based on infrared spectroscopy and density functional theory calculations. Polymer 2010, 51, 2597-2610.

(22) This estimation is made using Boltzmann distribution and corresponding ground state energies ${ }^{13}$.

(23) Veytsman, B. A. Are lattice models valid for fluids with hydrogen bonds? J. Phys. Chem. 1990, 94, 8499-8500.

(24) Coggeshaci, N. N.; Saier, E. L.; Coggeshall, N. N. Infrared Absorption Study of Hydrogen Bonding Equilibria. J. Am. Chem. Soc. 1951, 73, 5414-5418.

(25) Coleman, M. M.; Yang, X.; Painter, P. C.; Graf, J. F. Equilibrium Constants and the Prediction of Phase Behavior for Phenoxy Blends with Aliphatic Polyesters. Macromolecules 1992, 25, 4414-4424. 
(26) Mirkin, N. G.; Krimm, S. Structural dependence of NH stretch mode frequency shifts in amide and peptide systems. J. Phys. Chem. A 2004, 108, 5438-5448.

(27) Myshakina, N. S.; Ahmed, Z.; Asher, S. A. Dependence of amide vibrations on hydrogen bonding. J. Phys. Chem. B 2008, 112, 11873-11877.

(28) Galan, J. F.; Germany, E.; Pawlowski, A.; Strickland, L.; Galinato, M. G. I. Theoretical and Spectroscopic Analysis of N,N'-Diphenylurea and N,N'-Dimethyl-N,N'diphenylurea Conformations. J. Phys. Chem. A 2014, 118, 5304-5315.

(29) Lu, J.-F.; Zhou, Z.-Y.; Wu, Q.-Y.; Zhao, G. Density functional theory study of the hydrogen bonding interaction in formamide dimer. THEOCHEM 2005, 724, 107-114.

(30) Esrafili, M. D.; Behzadi, H.; Hadipour, N. L. 14N and $17 \mathrm{O}$ electric field gradient tensors in benzamide clusters: Theoretical evidence for cooperative and electronic delocalization effects in N-H.. O hydrogen bonding. Chem. Phys. 2008, 348, 175-180.

(31) In order to get this solution, we first introduce a function $f(z)=x g(x)$ and get the equation $x f^{\prime}-2 f f^{\prime}-2 f=0$. Then we go to the inverse function $x(f)$ for which $2 f x^{\prime}-x+2 f=0$. This equation has the solution $x=-2 f+A \sqrt{f}$. Finally, we solve the quadratic equation with respect to $f$.

(32) The On-Line Encyclopedia of Integer Sequences, published electronically at https://oeis.org, May 2018.

(33) Smaller values of the AICc parameter correspond to a better quality of fit. Usually, it is assumed that a difference between the AICc parameters of two models of more than 2 is significant and more than 6 is strong. See the supporting information for further details.

(34) Akaike, H. In Selected Papers of Hirotugu Akaike; Parzen, E., Tanabe, K., Kitagawa, G., Eds.; Springer New York: New York, NY, 1998; pp 199-213. 
(35) Sugiura, N. Further analysts of the data by Akaike's information criterion and the finite corrections. Commun. Stat. Theory Methods 1978, 7, 13-26.

(36) The model 2 is not considered here because of two reasons: (1) The validity of noncyclic approximation for model 2 is restricted to very low association constants or very low concentrations of acrylamide. So in this case it would be more interesting first to take into account the possibility of formation of cycles; (2) Another reason is that if we consider two parameter extension of non-cyclic approximation for model 2 we cannot find expressions in the closed form. It is possible to do it numerically, by means of solution of the differential equation for the generating function (similar to equation 13), but it is out the scope of the present paper.

(37) Kobko, N.; Dannenberg, J. J. Cooperativity in amide hydrogen bonding chains. Relation between energy, position, and H-bond chain length in peptide and protein folding models. J. Phys. Chem. A 2003, 10\%, 10389-10395.

(38) Hayashi, M.; Noro, A.; Matsushita, Y. Highly Extensible Supramolecular Elastomers with Large Stress Generation Capability Originating from Multiple Hydrogen Bonds on the Long Soft Network Strands. Macromol. Rapid Commun. 2016, 37, 678-684.

(39) Yang, J. X.; Fan, B.; Li, J. H.; Xu, J. T.; Du, B. Y.; Fan, Z. Q. Hydrogen-BondingMediated Fragmentation and Reversible Self-assembly of Crystalline Micelles of Block Copolymer. Macromolecules 2016, 49, 367-372.

(40) Song, D. P.; Lin, Y.; Gai, Y.; Colella, N. S.; Li, C.; Liu, X. H.; Gido, S.; Watkins, J. J. Controlled Supramolecular Self-Assembly of Large Nanoparticles in Amphiphilic Brush Block Copolymers. J. Am. Chem. Soc. 2015, 137, 3771-3774.

(41) Jeong, G.; Yu, D. M.; Mapas, J. K. D.; Sun, Z.; Rzayev, J.; Russell, T. P. Realizing 5.4 nm Full Pitch Lamellar Microdomains by a Solid-State Transformation. Macromolecules $\mathbf{2 0 1 7}, 50,7148-7154$. 
(42) Kwak, J.; Mishra, A. K.; Lee, J.; Lee, K. S.; Choi, C.; Maiti, S.; Kim, M.; Kim, J. K. Fabrication of Sub-3 nm Feature Size Based on Block Copolymer Self-Assembly for Next-Generation Nanolithography. Macromolecules 2017, 50, 6813-6818.

(43) Leibler, L. Theory of microphase separation in block copolymers. Macromolecules $\mathbf{1 9 8 0 ,}$ 13, $1602-1617$.

(44) For the case of alcohols, it was shown by Painter and Coleman that the association constants measured for a monomer can be used to describe association in polymer systems. In order to do this, the association constants should be rescaled to the molar volume of a polymer segment, so that

$$
K_{i}^{\text {polymer }}=\frac{v_{\text {acrylamide }}}{v_{\text {segment }}} K_{i}^{\text {acrylamide }}
$$

It is worth mentioning that association constants cannot be measured directly for polymers by infrared spectroscopy, for two reasons. The first is that hydrogen bonding polymers are not soluble in "inert" solvents that do not have specific interactions with the polymer. The second reason is that, since the hydrogen-bonding segments are connected by covalent bonds, all hydrogen-bonding segments have hydrogen-bonding neighbors even at infinite dilution, which means that the polymer segments are not randomly mixed.

(45) Morse, D. C.; Chung, J. K. On the chain length dependence of local correlations in polymer melts and a perturbation theory of symmetric polymer blends. J. Chem. Phys. 2009, 130, 224901.

(46) Lodge, T. P.; McLeish, T. C. B. Self-concentrations and effective glass transition temperatures in polymer blends. Macromolecules 2000, 33, 5278-5284.

(47) Painter, P. C.; Veytsman, B.; Kumar, S.; Shenoy, S.; Graf, J. F.; Xu, Y.; Cole- 
man, M. M. Intramolecular Screening Effects in Polymer Mixtures . 1. HydrogenBonded Polymer Blends. Macromolecules 1997, 30, 932-942.

(48) Lefèvre, N.; Daoulas, K. C.; Müller, M.; Gohy, J. F.; Fustin, C. A. Self-assembly in thin films of mixtures of block copolymers and homopolymers interacting by hydrogen bonds. Macromolecules 2010, 43, 7734-7743.

(49) Zhang, X.; Lin, J.; Wang, L.; Zhang, L.; Lin, J.; Gao, L. Supramolecular assembly of diblock copolymer blends with hydrogen-bonding interactions modeled by Yukawa potentials. Polymer 2015, 78, 69-80. 